\title{
Feature-guided waves for monitoring adhesive shear modulus in bonded stiffeners
}

Z. Fan $^{1}$, M. Castaings ${ }^{2}$, M. J. S. Lowe ${ }^{1}$, C. Biateau ${ }^{2}$, and P. Fromme ${ }^{3}$

${ }^{1}$ Department of Mechanical Engineering, Imperial College London, SW7 2AZ, UK

${ }^{2}$ Univ. Bordeaux, I2M, UMR 5295, F-33400 Talence, France.

CNRS, I2M, UMR 5295, F-33400 Talence, France.

Arts et Métiers ParisTech, I2M, UMR 5295, F-33400 Talence, France.

${ }^{3}$ Department of Mechanical Engineering, University College London, WC1E 7JE, UK

\begin{abstract}
Adhesively bonded stiffeners are employed in aerospace applications to increase structural stiffness. The potential of feature-guided wave modes for the verification of adhesion and curing state in difficult-to-access regions has been investigated. The properties of guided wave modes propagating along a T-shaped stiffener bonded to an aluminium plate were calculated using the Semi-Analytical Finite Element (SAFE) method. Feature-guided modes dominated by shearing motion were identified to be well suited, with energy concentrated at the stiffener and bond line, limiting energy radiation into the plate and thus maximising inspection length. The influences of the bond line stiffness and thickness on the guided wave behaviour were investigated using SAFE and 3D Finite Element calculations, and found to be significant. Experiments were conducted to measure the properties of the guided waves during the curing of an epoxy joint attaching a stiffener to a plate. The feature-guided mode was excited using a piezo-electric shear transducer and measured using a laser interferometer. The measured phase speed changed significantly during curing. The frequency dependency was found to match well with the SAFE calculations for a variation of the shear (Coulomb) modulus of the adhesive. The potential of the featureguided shear wave mode for bond line inspection and monitoring has been shown and the choice of guided wave mode and frequency range for good sensitivity to the bond line state discussed.
\end{abstract}

Keywords: Guided Ultrasonic Waves, Bond Line Monitoring, Feature-Guided Wave, Stiffener 


\section{Introduction}

Stiffeners are widely used in aircraft structures. Typically they participate in a complex structural assembly with the aircraft skin and the airframe, to provide both strength and stiffness. Adhesive bonding is a very attractive means of fixing the skin to the stiffener, a continuous bond here providing good stiffness and force transfer with relatively low stresses. The adhesive bonds between skin and stiffener are thus important to the performance of the whole structure [1], so the quality of the adhesive bond has to be verified. Typical problems which would need to be detected include poor adhesion of the adhesive material to the adherends and inadequate strength of the adhesive material itself caused by incorrect curing [2]. It has been shown that the speed of bulk waves in the adhesive material, which is dependent primarily on the stiffness of the material, may be used to infer the state of cure of the adhesive [3]. This has motivated studies to monitor curing using ultrasonic techniques [4-7].

If the bond line geometry is continuous in at least one dimension then guided elastic waves can be an attractive means of interrogating the bond. Conceptually, it is expected that a guided wave which propagates along a length of the bond may be exploited to be particularly sensitive to the properties of the bond. This idea has been pursued in many studies of adhesive joints of parallel plates using Lamb waves and interface waves [4, 810], and is reviewed for the different possibilities of guided waves in [10]. In a slightly different configuration, it has also been shown that guided waves dominant in a parent material can be used to interrogate the attachment of another material, such as a spar on a skin [1] or repair patch on a skin [11].

An interesting further development is to consider a wave which is guided along a feature, in which case a trapped mode may retain its energy at the feature, so enabling long distance propagation. Trapped modes may exist in many topics of physics and have been previously investigated: the circumstances that can support the existence of trapped elastic modes include local deformations of the waveguide geometry such as bends, thickening or thinning; the nature of these phenomena has been investigated by Craster and co-workers, see for example [12]. The ideal case here would be a mode whose phase speed is below that of any of the modes which may propagate in the parent body, so that there is no possibility of energy radiating from the feature; this we might call a perfectly-trapped mode. But other cases, of partially-trapped modes, in which some energy radiates yet the mode remains dominant at the feature may also be interesting. This idea has been studied successfully for the case of a butt weld between two plates. The geometrical profile of the weld cap, presenting a local increase in the thickness of the plate, allows the existence of just such modes. This was found first by observation of a compression-like mode [13], which is dispersive, partially-trapped at low frequencies, and perfectly-trapped at high frequencies. This geometry was then investigated by model and further experiments, showing the existence of both the compression-like mode and a perfectly-trapped shear-like mode with very little dispersion $[14,15]$. The shear-like mode is slower than any other mode at all frequencies, and so does not leak any energy into the adjacent plates. The possibility of using these waves to inspect the weld material and the material in the nearby heat-affected zone (HAZ) was later demonstrated.

In the present work, we consider the potential to exploit feature-guided waves to inspect adhesive bonds between stiffeners and skins, in an arrangement typical of aircraft construction. A wave which is trapped in the stiffener region (including the stiffener, the adhesive bond and the zone of the skin that is immediately underneath the bond) may be 
useful to perform rapid inspection along a long length of the stiffener with little or no loss of energy into the skin to which it is attached. In principle this approach could be used to detect any number of candidate defects in the stiffener. In this study we consider the particular case of the inspection of the layer of adhesive which attaches the stiffener to the skin. As discussed above, the strength of the adhesive is understood to be related to its state of cure, and this is shown by its stiffness. The stiffness of the joint is also affected by its thickness. Therefore, if properties of feature-guided waves in the stiffener are found to be affected by the stiffness of the adhesive layer, then there would be a basis to use the guided waves for the inspection of the state of cure of the adhesive and/or of its thickness. This could be useful for fast inspection or for inspecting components where access is difficult.

The paper starts with a summary of modelling work, to identify and understand the guided wave modes that can propagate along a stiffener attached to an aircraft skin. This has been done using the so-called SAFE (Semi-Analytical Finite Element) method which employs a two-dimensional Finite Element (FE) modal approach to determine the propagating modes and identify suitable guided wave modes. A three-dimensional FE approach has been employed to model the wave propagation, validate the predicted dispersion behaviour, and to demonstrate the proposed signal analysis which is based on a two-dimensional Fourier transform. The studies investigate the effect of the properties of the adhesive layer, found by parametric variations in the models, on the wave propagation characteristics of the chosen feature-guided shear wave mode, especially the phase speed dispersion curves. Following that, the paper describes the experimental work, involving the measurement of the speed of the feature-guided waves during the curing of the adhesive. The results of the modelling and experimental work are compared, to assess the feasibility of determining the properties of the adhesive layer from guided wave measurements. Recommendations are given for the choice of feature-guided wave modes and frequency range.

\section{SAFE Modelling}

The first task in the investigation was to identify and understand the guided waves which may exist in a stiffener attached to a skin. Whereas the calculation of guided waves in regular geometric shapes is straightforward using analytical techniques, it is necessary to resort to numerical methods to study waves in complex geometries such as the stiffener. A popular method for prismatic waveguides is the so-called SAFE (Semi-Analytical Finite Element) method, which uses Finite Elements to discretise the cross-section of the waveguide, combined with an analytical description of the behaviour in the direction along the waveguide, thus limiting the FE model to two dimensions [14-20]. This has been shown in the literature to be particularly useful for some well-known problems of irregularly-shaped waveguides such as railway lines [16, 17]. In most cases SAFE analysis has been deployed using specific programming, or advanced use of a flexible commercial code such as COMSOL [21], but it can be possible to perform SAFE modelling very simply, using standard data inputs without programming, in popular proprietary commercial FE codes [19].

The study presented here made use of the implementation reported by Predoi et al [22], with the extension for complex propagating modes reported by Castaings and Lowe [15]. The latter is necessary for the solutions for partially-trapped modes, to enable the model to include waves which leak energy from the guiding feature (the stiffener) into radiating waves in the attached plate (the skin). The method, applied to the stiffener problem, is summarised in the following paragraphs. 
Harmonic guided waves propagating along the stiffener $\left(\mathrm{x}_{3}\right.$ axis) are considered. There is no geometric variation of the cross-section (shown in Fig. 1) along $\mathrm{x}_{3}$, so the behaviour in this direction is simple and can be written in analytical form. Thus the displacements vector $\mathrm{u}_{\mathrm{r}}$ in the waveguide can be written as:

$$
\boldsymbol{u}_{r}\left(x_{1}, x_{2}, x_{3}, t\right)=\boldsymbol{U}_{r}\left(x_{1}, x_{2}\right) e^{I\left(k x_{3}-\omega t\right)} \quad I=\sqrt{-1},
$$

in which $k$ is the complex wavenumber along the axial direction $\left(x_{3}\right)$ of the waveguide, $\omega$ is the angular frequency, $t$ is the time variable and the subscript $r=1,2,3$. The real part of $k$ describes the harmonic wave propagation while the imaginary part describes its attenuation, which may be caused by leakage of radiating waves into the adjacent plate, or by material damping if this is included in the material description. The function $U_{r}$ represents the behaviour in the cross-section of the waveguide $\left(\mathrm{x}_{1}-\mathrm{x}_{2}\right.$ plane), for which the geometry is irregular, such that it is incorporated in the model by a two-dimensional FE discretization. Full details of how this is done are given in $[15,22]$.

For chosen values of angular frequency $\omega$ eigenvalues of the complex wavenumber $k$ are found, that represent guided wave modes propagating along the stiffener, while possibly radiating energy into the plate. For the construction of dispersion curves, each solution at a chosen frequency reveals the wavenumbers, attenuations and mode shapes of all of the possible modes at that frequency; the full dispersion curve spectrum is then found by repeating the eigenvalue solutions over the desired range of frequencies. The formalism for the eigenvalue problem was implemented in the commercial FE code COMSOL [21].

Figure 1 shows a schematic of the cross-section of the stiffener, adhesive and part of the plate, which were discretised in the SAFE model. The 2D model included the T-shaped aluminium stiffener (size: $20 \mathrm{~mm}$ x $20 \mathrm{~mm}, 1.5 \mathrm{~mm}$ thickness, $\rho=2700 \mathrm{~kg} / \mathrm{m}^{3}$, $C_{11}=107.84 G P a, C_{66}=26.54 G P a$ ), the adhesive bond (several thicknesses ranging from 0.5 to $1.0 \mathrm{~mm}$ and material properties to simulate imperfect curing were considered in different models, $\rho=1050 \mathrm{~kg} / \mathrm{m}^{3}, C_{11}=(5.6+0.5 i) G P a, C_{66}=m(1+0.1 i) G P a$, where $\mathrm{m}$ varies from 0.02 to 0.5 ) and the aluminium plate (thickness $3 \mathrm{~mm}$, same material properties as stiffener). Absorbing regions of length $0.8 \mathrm{~m}$ were included on both sides of the $2 \mathrm{~m}$ wide aluminium plate, to avoid standing wave modes and to allow proper representation of radiating leaking waves if present. As described in reports of previous work with absorbing regions [23, 24], the absorbing region had the same mass density and elastic properties as the medium of interest, but its viscoelastic coefficient gradually increased with the distance away from the region of interest. Continuity of displacements and stresses was imposed at the internal borders between the stiffener, the adhesive layer and the aluminium plate. Stress-free conditions were chosen at all outer limits of the domain. The whole geometry was meshed by approximately 1500 quadratic Lagrange triangular elements, the exact number depending on the bond line thickness) with side lengths comprised between 0.2 and $4 \mathrm{~mm}$. These elements were automatically generated by the software, and were finer in the stiffener and adhesive zones than in the adjacent plates. The various element sizes were chosen to ensure good spatial representation of the field in the cross-section. A series of numerical trials indicated a good convergence of the FE solutions. The number of degrees of freedom was approximately 25000, and the equation was solved for 56 frequencies in the range from 10 to $120 \mathrm{kHz}$. 
For each frequency, multiple solutions for $k$ were obtained, including the wanted waveguide modes, with behaviour concentrated in the stiffener region, but also unwanted resonances of the whole domain that had to be separated from the collection and discarded. Potential feature-guided wave modes were identified from the axial component of the timeaveraged energy flow (Poynting vector [25]), in a similar manner as was deployed in previous SAFE studies of leaky wave problems [14, 15]. Figure 2 shows the mode shape of the energy flow of a suitable mode (solid line) with energy flow concentrated close to the stiffener, and no energy flow in the absorbing region. The dash-dotted lines depict unwanted modes at the same frequency, being resonances of the whole cross-section.

\section{Identification of Feature-Guided Shear Waves along Stiffener}

From the SAFE calculations detailed above, the dispersion relation in the low frequency range up to $120 \mathrm{kHz}$ was calculated for assumed typical values of $0.5 \mathrm{GPa}$ shear modulus of the adhesive with a layer thickness of $0.5 \mathrm{~mm}$. Only feature-guided modes with a timeaveraged axial energy flow concentrated close to the stiffener were considered. Modes dominated by longitudinal, bending and shear deformations were identified and the energy distribution and mode shapes were characterized. Shear modes were chosen as the most suitable type, as they showed limited dispersion in the frequency range of interest, good sensitivity to changes in the bond layer properties and negligible leakage in the side plates.

Figure 3 shows the dispersion diagram for the feature-guided shear modes (identified from processing of the SAFE calculations and marked as modes 1, 2, and 3) with the $\mathrm{SH}_{0}$ mode in the aluminium plate plotted for comparison. It can be observed that in the frequency range of interest there are three shear modes. The phase speed of the feature-guided wave modes is below the $\mathrm{SH}_{0}$ speed in the plate $(\sim 3135 \mathrm{~m} / \mathrm{s})$, one of the necessary conditions to obtain no leakage of the shear mode into $\mathrm{SH}_{0}$ plate waves [15]. The mode shapes for phase velocities close to $3000 \mathrm{~m} / \mathrm{s}$ are illustrated in Figure 4 at frequencies of $15 \mathrm{kHz}$ for mode 1 , $40 \mathrm{kHz}$ for mode 2 , and $100 \mathrm{kHz}$ for mode 3 . The colour in this figure corresponds to the energy flow along $\mathrm{x}_{3}$ and the shear motion can be seen from the arrows showing the lateral motion in the cross-section. Different relative motions of the plate, bond line and stiffener can be seen for the different modes at the shown frequencies. The upper part of the stiffener has a bending motion characterized by the ratio of the wavelength to the stiffener height. In general good energy concentration close to the stiffener and in the bond line was observed, with a variation for the different shear modes and excitation frequencies.

\section{Finite Element Modelling in 3D}

In a separate model study, the propagation of the feature-guided shear wave modes was simulated using a 3D FE model in the proprietary code ABAQUS Explicit [26]; the model is shown in outline in Figure 5. The model was set up to represent the same stiffener and bond line cross section as had been studied with the SAFE model, (Fig. 1). The $3 \mathrm{~mm}$ thick aluminium plate to which the stiffener was bonded was modelled with a width of $1 \mathrm{~m}$ and length of $1.2 \mathrm{~m}$. The plate was discretised using linear brick elements (C3D8R) of dimensions $\Delta \mathrm{x}_{1}=\Delta \mathrm{x}_{3}=2 \mathrm{~mm}, \Delta \mathrm{x}_{2}=1 \mathrm{~mm}$. Smaller elements with size $\Delta \mathrm{x}_{1}=\Delta \mathrm{x}_{2}=\Delta \mathrm{x}_{3}=$ $0.5 \mathrm{~mm}$ were used for the stiffener, and $\Delta \mathrm{x}_{1}=\Delta \mathrm{x}_{3}=0.5 \mathrm{~mm}, \Delta \mathrm{x}_{2}=0.25 \mathrm{~mm}$ for the bondline. The plate, adhesive bone line and the stiffener were assembled using surface-tosurface tie constraints. Absorbing regions $[23,24]$ of width $0.4 \mathrm{~m}$ were implemented at the lateral extremes of the model, parallel to the stiffener, to avoid reflections from the edges of the plate. 
Forcing in the $\mathrm{x}_{1}$ direction was introduced at the stiffener front edge to excite the sheardominated feature-guided modes. The specification of the excitation was chosen to represent the setup for the experimental study described below. The excitation shear force was applied with equal amplitude on the nodes in a $1 \mathrm{~mm}$ by $1 \mathrm{~mm}$ square section at the conjunction of the beam and base of the stiffener. Thus an excitation pulse was defined, comprising a 5 cycle tone burst convolved with a Hanning window. The centre frequency of the tone burst was set as 40 and $100 \mathrm{kHz}$ respectively for two separate runs of the model. The mesh refinement in the propagation direction was therefore a minimum of 11 elements per wavelength in the coarsest case.

Explicit time integration was used, and the time step was chosen as $50 \mathrm{~ns}$ to adhere to the usual stability criterion [27]. The amplitude in the $\mathrm{x}_{1}$ direction of the propagating wave modes was monitored along the length of the stiffener. The line of monitoring nodes was located on the top of the stiffener base as shown in Figure 5 and consists of 301 nodes with a step size of $1 \mathrm{~mm}$, starting $500 \mathrm{~mm}$ from the excitation location. The set of time traces was processed using a 2D Fast Fourier transform (FFT) [28] with zero padding in the $\mathrm{x}_{3}$ direction to improve the resolution in the wavenumber domain. Based on the amplitude maxima at each frequency, the wavenumber and from this the phase speed of the dominant shear mode in the signals were determined.

The illustration in Figure 5 includes results of the model for an excitation frequency of 40 $\mathrm{kHz}$ at an instant in time during the solution. The colour contours show the magnitude of the resultant displacement, from which two wave packets can be seen propagating along the stiffener. These two modes correspond to the shear modes 1 and 2 marked in Figure 3 at 40 $\mathrm{kHz}$. The arrival times match the predicted group speeds $(1050 \mathrm{~m} / \mathrm{s}$ for mode 1 and 2750 $\mathrm{m} / \mathrm{s}$ for mode 2 ), with mode 2 propagating significantly faster than mode 1 . Both modes appear to be trapped at the stiffener, although mode 2 radiates very limited energy in the $\mathrm{A}_{0}$ mode in the plate close to the stiffener. This is similar to the localisation seen in the study of the weld-guided waves in [14].

The 3D FE simulations were compared carefully with the results of the SAFE analysis. The feature guided shear wave propagation along the stiffener was recorded, and the wavenumber of the dominant shear mode extracted by 2D FFT processing for each frequency. The phase speed was compared to the dispersion diagrams obtained from the SAFE calculations. A range of model results was computed, covering cases with shear modulus of the bond material from a very low value $(0.02 \mathrm{GPa})$ up to the nominal value of $0.5 \mathrm{GPa}$, intended to cover the range of properties of the adhesive from uncured through to cured conditions. The results of this comparison are shown in Figure 6, for the shear guided wave modes between $30 \mathrm{kHz}$ and $120 \mathrm{kHz}$. The results show good agreement between the predictions from the SAFE model and those from the 3D FE model.

\section{Discussion of Findings from the Models}

The reduced shear modulus of the bond layer results in a significant drop of the phase speed for modes 2 and 3 (Fig. 6). The drop for mode 3 is about $10 \%$ for the considered variation of shear modulus and the shape of the dispersion curve does not change significantly. For mode 2, especially below $60 \mathrm{kHz}$, a more significant change of the phase speed of more than $20 \%$ is predicted as well as a change of shape of the dispersion curves, corresponding to a higher sensitivity of this mode to changes in the bond layer. For mode 1 a significant 
variation of the phase speed is only predicted around $20 \mathrm{kHz}$, however that is very much at the lower end of the useable frequency range (for the investigated geometry and excitation). For the modes above $30 \mathrm{kHz}$ the phase speed extracted from the 3D FE simulations matches well to the predicted dispersion curves from the SAFE calculations and the uncertainty level is lower than the predicted changes due to the variation of the shear modulus of the bond layer. This suggests a good sensitivity for the monitoring of the bond line properties using the feature-guided shear wave propagating along the stiffener. Nevertheless, it should be remembered that this is in ideal conditions with optimally obtained noise-free data.

In practical applications the adhesive bond layer thickness cannot always be well controlled. The influence of a variation of the thickness of the bond layer between $0.5 \mathrm{~mm}$ and $1.0 \mathrm{~mm}$ on the dispersion curves was predicted from SAFE calculations for a shear modulus of $0.2 \mathrm{GPa}$. Shown in Figure 7 are the dispersion curves for the same frequency range of the three dominant shear guided modes. For a variation of the bond line thickness by a factor of two a smaller influence $(<10 \%)$ on the phase speed and shape of the dispersion curves can be observed over the complete frequency range. Mode 1 shows almost no influence of the bond line thickness. For mode 3 it might be difficult to distinguish between a variation of the bond line thickness and material properties if both are uncertain, as this mode shows a similar behaviour as seen in Figure 6, although with a smaller change. On the other hand, the behaviour of mode 2 between $30 \mathrm{kHz}$ and $60 \mathrm{kHz}$ could in principle allow for a monitoring of both the bond line property and the thickness, as they have different influences on this mode. A realistic variation of the thickness influences only the phase speed by less than $10 \%$ but not the overall shape of the dispersion curve, while a lower shear modulus due to incomplete curing leads to a change in the shape of the dispersion curve as well as a larger change of the phase speed (Fig. 6). However, these distinctions of shapes of the dispersion curves could be difficult to achieve in practice when the measurements are affected by noise and other uncertainties. Therefore it is safer to conclude for practical exploitation just that the sensitivity is sufficient to allow for the monitoring of the bond line thickness for known shear modulus of the adhesive, or vice versa.

\section{Experimental Setup}

An experimental programme was conducted in order to validate the findings of the model studies and determine whether it could be practical to exploit the observed sensitivity to measure the properties of the bondline. A T-shaped aluminium stiffener (cross-section: 20 $\mathrm{mm} \times 20 \mathrm{~mm}, 1.4 \mathrm{~mm}$ thickness, length: $660 \mathrm{~mm}$ ) was bonded adhesively to a $3 \mathrm{~mm}$ thick aluminium plate (size $500 \mathrm{~mm}$ x $660 \mathrm{~mm}$ ). An epoxy-based adhesive was chosen (DGEBA-PAMAM adhesive system) that cures over a long time, depending on the substrates, the bond thickness and the ambient temperature. The curing behaviour of the adhesive over time had been monitored in an independent study using bulk ultrasonic waves [29], providing the shear stiffness over time with a final value of approximately 0.5 $\mathrm{GPa}$ after 52 hours. The bond line thickness was measured using a dial gauge as approximately $1 \mathrm{~mm}$, with a variation of $+/-0.1 \mathrm{~mm}$ along the length of the stiffener.

A piezoelectric shear transducer was used to generate the feature-guided shear wave, as shown in Figure 8. The transducer was positioned at one end of the stiffener base, transmitting the shear motion via the SWC shear wave couplant supplied by Sofranel. The transducer was driven by a $150 \mathrm{~V}$ amplitude chirp waveform to produce motion in the frequency range from 10 to $100 \mathrm{kHz}$ (down to $-6 \mathrm{~dB}$ ). A Polytec laser interferometer (OFV 
505/5000) was moved along the stiffener using a scanning rig to measure the transverse motion of the upper part of the stiffener. Retro-reflective tape was placed at one side of the stiffener for optimum reflection of the laser beam, to improve the signal to noise ratio. Time traces of the out-of-plane displacement were measured every $5 \mathrm{~mm}$ over a length of $270 \mathrm{~mm}$, using 150 averages.

The wave propagation was measured regularly during the curing process over several days. Two typical time traces measured $170 \mathrm{~mm}$ from the excitation are shown in Figure 9 at $3 \mathrm{~h}$ from the start of curing and at $164 \mathrm{~h}$ at the end of the curing process. The incident, multimodal wave pulse can be clearly seen from 0.2 to 0.6 microseconds, with the reflection from the end of the stiffener visible after 0.9 microsecond propagation time. A significant change in the pulse due to the curing of the adhesive can be observed as a change in amplitude, shape and arrival time of the components between the two time signals. A time window was applied to suppress the reflections using a cosine tapered window from $0 \mathrm{~ms}$ to $0.8 \mathrm{~ms}$. The measurements were processed using a 2D FFT with zero padding both in the space and time domain, as was discussed earlier in the context of the 3D FE simulations, but with slightly different settings. From the wave number - frequency diagram the 3 dominant shear modes were identified as local maxima, and the phase speed dispersion curve of each mode for the frequency range up to $120 \mathrm{kHz}$ was calculated.

\section{Comparison of experimental results with simulations}

The measured phase speed dispersion curves in the frequency range up to $120 \mathrm{kHz}$ are shown in Fig. 10 for three time steps during the curing of the adhesive. The time steps at $3 \mathrm{~h}, 8 \mathrm{~h}$, and $164 \mathrm{~h}$ from the mixing of the adhesive show, as predicted, a general increase in the phase speed of the shear modes and a change in the shape of the dispersion curves. The best fit of the SAFE predictions to these results was found by varying the modulus of the adhesive within its permissible range up to the value of $\mathrm{G}=0.5 \mathrm{GPa}$ (fully cured), while holding the bondline thickness to the known value of $1 \mathrm{~mm}$. The best fit results were at $0.02 \mathrm{GPa}$ for $3 \mathrm{~h}$ of curing time, $0.07 \mathrm{GPa}$ for $8 \mathrm{~h}$ curing time and $0.5 \mathrm{GPa}$ for $164 \mathrm{~h}$ curing time ( 7 days). The elastic modulus of this adhesive material had previously been characterised by independent means (bulk ultrasonic measurement and wedge test) at various stages of cure as part of a separate investigation [29]. Our results indicate a slower curing than that in the separate investigation of [29]. In that case the shear modulus after $3 \mathrm{~h}$ of curing was about $0.22 \mathrm{GPa}$, and after $8 \mathrm{~h}$ of curing was about $0.42 \mathrm{GPa}$. The curing rate of adhesive is very sensitive to temperature and thus the configuration, which differed significantly compared to [29] (thickness $0.16 \mathrm{~mm}$, assembled materials aluminium and Carbon Epoxy composite). However, the shear modulus for the fully cured state matches well with the previous results of approximately $\mathrm{G}=0.5 \mathrm{GPa}$ after $145 \mathrm{~h}$.

It can be observed from Figure 10 that the measured and calculated dispersion curves show generally good agreement. As predicted from the SAFE simulations, for mode 3 above 60 $\mathrm{kHz}$ a smaller influence of the adhesive properties of about $10 \%$ change in the phase speed was measured, with no significant change in the shape of the dispersion curve. For the lowest shear modulus value $(\mathrm{G}=0.02 \mathrm{GPa})$ and thick bondline $(1.0 \mathrm{~mm})$ the dispersion curve of mode 3 matches the dispersion curve of the stiffener as the energy is localized only in the stiffener without coupling into the plate. Around $80 \mathrm{kHz}$ one can observe a drop-out of the experimentally measured phase speed curve as the mode is only excited weakly at that frequency (this was observed in the 2D FFT data). For shear guided wave mode 2 from 30 to $60 \mathrm{kHz}$, a change of about $20 \%$ of the phase speed with the curing of 
the adhesive was measured, with a distinctive change in the frequency dependency of the phase speed. The lowest shear guided wave mode above $30 \mathrm{kHz}$ showed almost no dependency on the curing state of the adhesive, as predicted from the numerical simulations. Around $20 \mathrm{kHz}$ a distinctive change in the phase speed can be observed, however that is too close to the lower practical limit of the inspection frequency due to the low frequency cut-off of the transducer.

It seems therefore that the best sensitivity of the shear guided waves is predicted for mode 2 between $30 \mathrm{kHz}$ and $60 \mathrm{kHz}$, and this is also verified from the experimental measurements, for which this was the most strongly excited mode (amplitude at least twice that of other modes at each frequency). For the investigated configuration it would be sufficient to concentrate on this mode and frequency range. For different configurations the optimum mode and frequency range can be predicted with sufficient accuracy solely by using the fast SAFE calculations of the cross-section without the need for full 3D FE modelling.

\section{Conclusions}

The propagation of feature guided waves along a stiffener adhesively bonded to a plate has been investigated using both simulations and experiments. The propagation characteristics of the wave modes along the stiffener bonded to the plate were predicted using efficient Semi Analytical Finite Element (SAFE) calculations. This model study identified the existence and properties of shear-dominated guided wave modes whose energy is concentrated along the stiffener and adhesive layer. They have limited dispersion and energy radiation into the plate, and thus may propagate long distances. The wave propagation of these modes was simulated using a 3D Finite Element (FE) model. The time traces of the waves were recorded at a series of locations along the stiffener, enabling a two-dimensional Fourier transform (2D FFT) analysis to be used to extract the wave speeds. Good agreement with the dispersion curves calculated using the SAFE method was found. The influence on the phase speed dispersion curves of bondline thickness and shear (Coulomb) modulus was evaluated. Significant influence of the variation of the shear modulus on the phase speed was observed between the uncured and the cured states. The shape of the dispersion curves was as well observed to change. Experiments were conducted during the slow curing of an epoxy adhesive, bonding a stiffener to the plate, with bond strength and stiffness increasing over time. The excited shear mode was measured using a laser interferometer and evaluated using a 2D FFT analysis in a similar manner as was used for the FE simulations. The measured phase speed changed significantly during curing, and it was shown that these values could be matched to speeds predicted by SAFE calculations for part-cured adhesive, for a realistic variation of the shear modulus of the adhesive layer. The potential of the feature guided shear wave modes for bond line inspection and monitoring has been shown. From the efficient SAFE calculations suitable feature guided wave modes and frequency range were well predicted, allowing the optimum design of the monitoring measurements.

\section{Acknowledgements}

The authors would like to thank Rescoll company (http://www.rescoll.fr/accueil.php) in Bordeaux, France, for the free supply of the adhesive components. 


\section{References}

1. L. F. Lanza di Scalea, H. Matt, I. Bartoli, S. Coccia, G. Par, and C. Farrar, "Health monitoring of UAV wing skin-to-spar joints using guided waves and macro fiber composite transducers", J. Int. Mat. Sys. Struct. 18, pp. 373-388, 2007.

2. R.B. Thompson and D.O Thompson, "Past experiences in the development of tests for adhesive bond strength", J. Adhesion Sci Technol, 5, pp. 583-599, 1991.

3. A.M. Lindrose, "Ultrasonic wave and moduli changes in a curing epoxy resin", Exp. Mech. 18, pp. 227-232, 1978.

4. S.I. Rokhlin, M. Hefets, and M. Rosen, "An ultrasonic interface wave method for predicting the strength of adhesive bonds", J. Appl. Phys. 52, pp. 2847-2851, 1981.

5. R. J. Freemantle and R. E. Challis, "Combined compression and shear wave ultrasonic measurements on curing adhesive", Meas. Sci. Techn. 9, pp. 1291-1302, 1998.

6. S. Dixon, D. Jaques, S. B. Palmer, and G. Rowlands, "The measurement of shear and compression waves in curing epoxy adhesives using ultrasonic reflection and transmission techniques simultaneously", Meas. Sci. Techn. 15, pp. 939-947, 2004.

7. R.E. Challis, F. Blarel, M.E. Unwin, J. Paul, and X. Guo, "Models of ultrasonic wave propagation in epoxy materials", IEEE Trans. UFFC 56, pp. 1225-1237, 2009.

8. A.K. Mal, P.C. Xu and Y. Bar-Cohen, "Analysis of leaky Lamb waves in bonded plates", Int. J. Engng. Sci. 27, pp. 779-791, 1989.

9. P.B. Nagy and L. Adler, "Nondestructive evaluation of adhesive joints by guided waves", J. Appl. Phys. 66, pp. 4658-4663, 1989.

10. M.J.S. Lowe and P. Cawley, "The applicability of plate wave techniques for the inspection of adhesive and diffusion bonded joints", J. NDE 13, pp. 185-199, 1994.

11. B. Le Crom and M. Castaings, "Shear horizontal guided wave modes to infer the shear stiffness of adhesive bond layers", J. Acoust. Soc. Am. 127, pp. 2220-2230, 2010.

12. J. Postnova and R.V. Craster, "Trapped modes in topographically varying waveguides", Wave Motion, 44, pp. 205-221, 2007.

13. J. P. Sargent, "Corrosion detection in welds and heat-affected zones using ultrasonic Lamb waves", Insight 48, pp. 160-167, 2006.

14. Z. Fan and M. J. S. Lowe, "Elastic waves guided by a welded joint in a plate", Proc. Roy. Soc. A 465, pp. 2053-2068, 2009.

15. M. Castaings and M. J. S. Lowe, "Finite element model for waves guided along solid systems of arbitrary section coupled to infinite solid media", J. Acoust. Soc. Am. 123, pp. 696-708, 2008.

16. L. Gavric, "Computation of propagative waves in free rail using a finite element technique," J. Sound Vib. 185, pp. 531-543, 1995.

17. V. Damljanovic and R. L. Weaver, "Propagating and evanescent elastic waves in cylindrical waveguides of arbitrary cross section," J. Acoust. Soc. Am. 115, 1572-1581, 2004.

18. I. Bartoli, A. Marzani, F. Lanza di Scalea, and E. Viola, "Modeling wave propagation in damped waveguides of arbitrary cross-section," J. Sound Vib. 295, pp. 685-707, 2006.

19. P. Wilcox, M. Evans, O. Diligent, M. J. S. Lowe, and P. Cawley, "Dispersion and excitability of guided acoustic waves in isotropic beams with arbitrary cross-section", Review of Progress in Quantum Nondestructive Evaluation, ed D.O. Thompson and D.E. Chimenti, AIP Conf. Proc. 21, pp. 203-210, 2002.

20. K. Jezzine and A. Lhemery, "Diffraction effects on ultrasonic guided waves radiated or received by transducers mounted on the section of the guide", Review of Progress in Quantum Nondestructive Evaluation, ed D.O. Thompson and D.E. Chimenti, AIP Conf. Proc. 25, pp. 134-141, 2006. 
21. COMSOL, User's Guide and Introduction. http://www.comsol.com/.

22. M. V. Predoi, M. Castaings, B. Hosten, and C. Bacon, "Wave propagation along transversely periodic structures", J. Acoust. Soc. Am. 121, pp. 1935-1944, 2007.

23. M. Castaings, C. Bacon, B. Hosten, and M. V. Predoi, "Finite element predictions for the dynamic response of thermo-viscoelastic material structures", J. Acoust. Soc. Am. 115, pp. 1125-1133, 2004.

24. P. Rajagopal, M. Drozdz, E.A. Skelton, M.J.S. Lowe, and R.V. Craster, "On the use of absorbing layers to simulate the propagation of elastic waves in unbounded isotropic media using commercially available Finite Element packages", NDT\&E Int. 51, pp. 3040, 2012.

25. B. A. Auld, "Acoustic fields and waves in solids", R.E. Krieger, Malabar, USA, 1990.

26. ABAQUS version 6.8, User's Guide and introduction. http://www.simulia.com/.

27. K.-J. Bathe, "Finite Element Procedures in Engineering Analysis", Prentice-Hall, Englewood Cliffs, New Jersey, USA, 1982.

28. D. Alleyne and P. Cawley, "A two-dimensional Fourier transform method for the measurement of propagating multimode signals", J. Acoust. Soc. Am., 89, pp. 1159$1168,1991$.

29. M. K. Budzik, B. Mascara, J. Jumel. M. Castaings, and M. E. R. Shanahan, "Monitoring of crosslinking of a DGEBA-PAMAM adhesive in composite/aluminium bonded joint using mechanical and ultra-sound techniques," Int J. Adhesion \& Adhesives 35, pp. 120-128, 2012. 


\section{Figures}

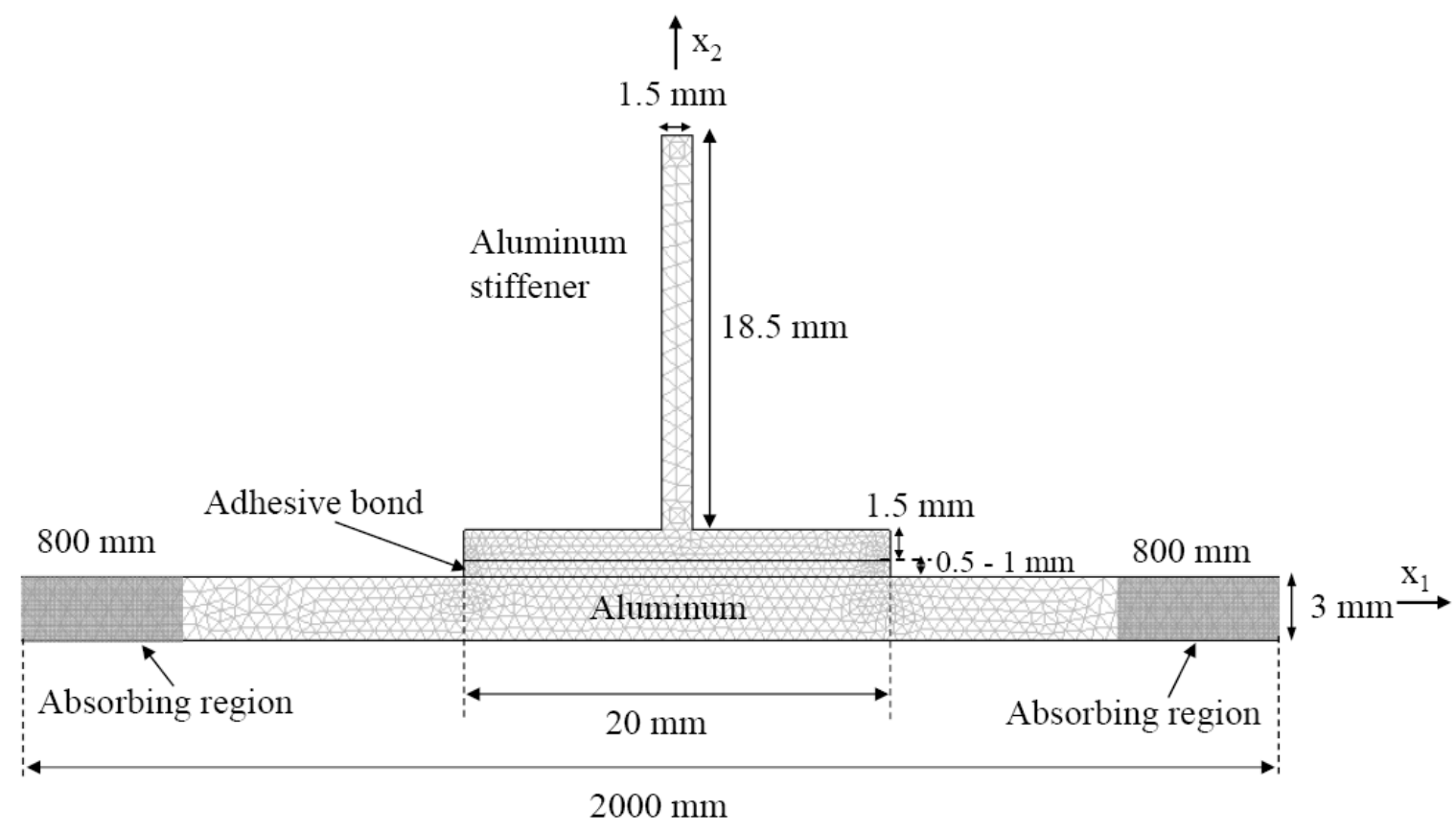

FIGURE 1. Schematic of SAFE model of aluminum plate with stiffener, adhesive bond layer, and absorbing regions shown (not to scale).

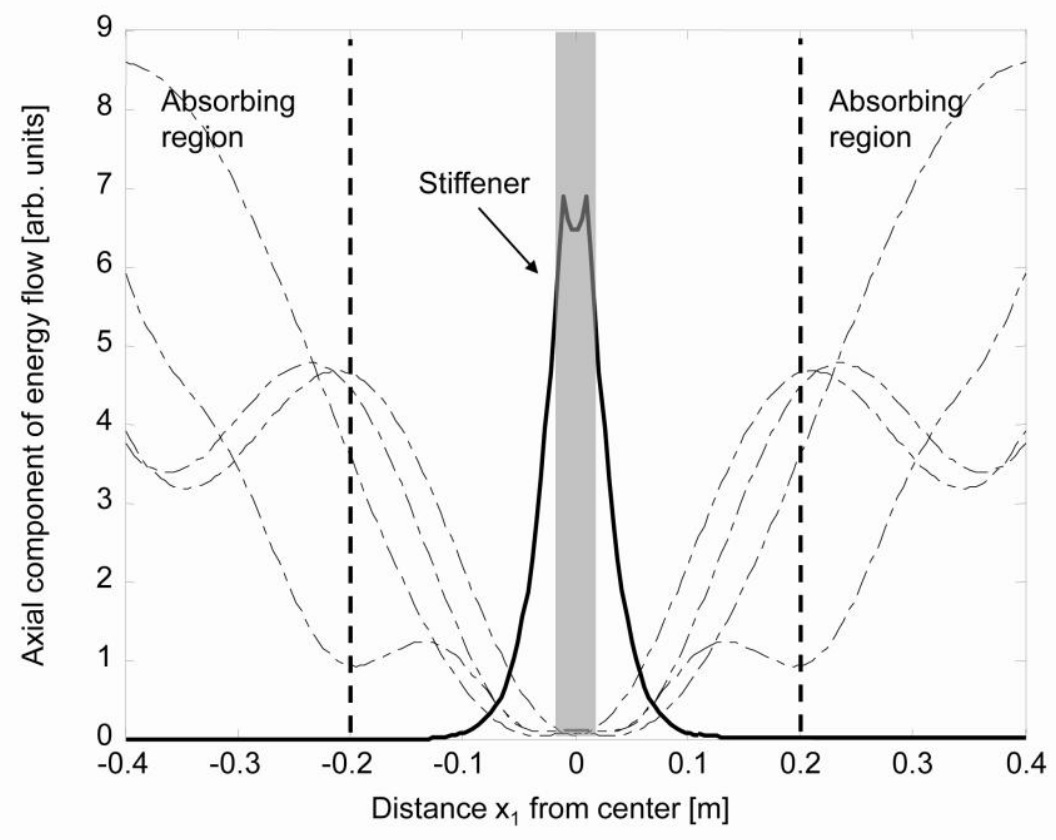

FIGURE 2. Axial component of energy flow in stiffened aluminum plate, calculated from SAFE model at $\mathrm{f}=40 \mathrm{kHz}$; suitable mode with energy concentrated around stiffener (solid line), unsuitable modes (dashdotted lines); boundary with absorbing regions (dashed lines) and stiffener (shaded) indicated. 


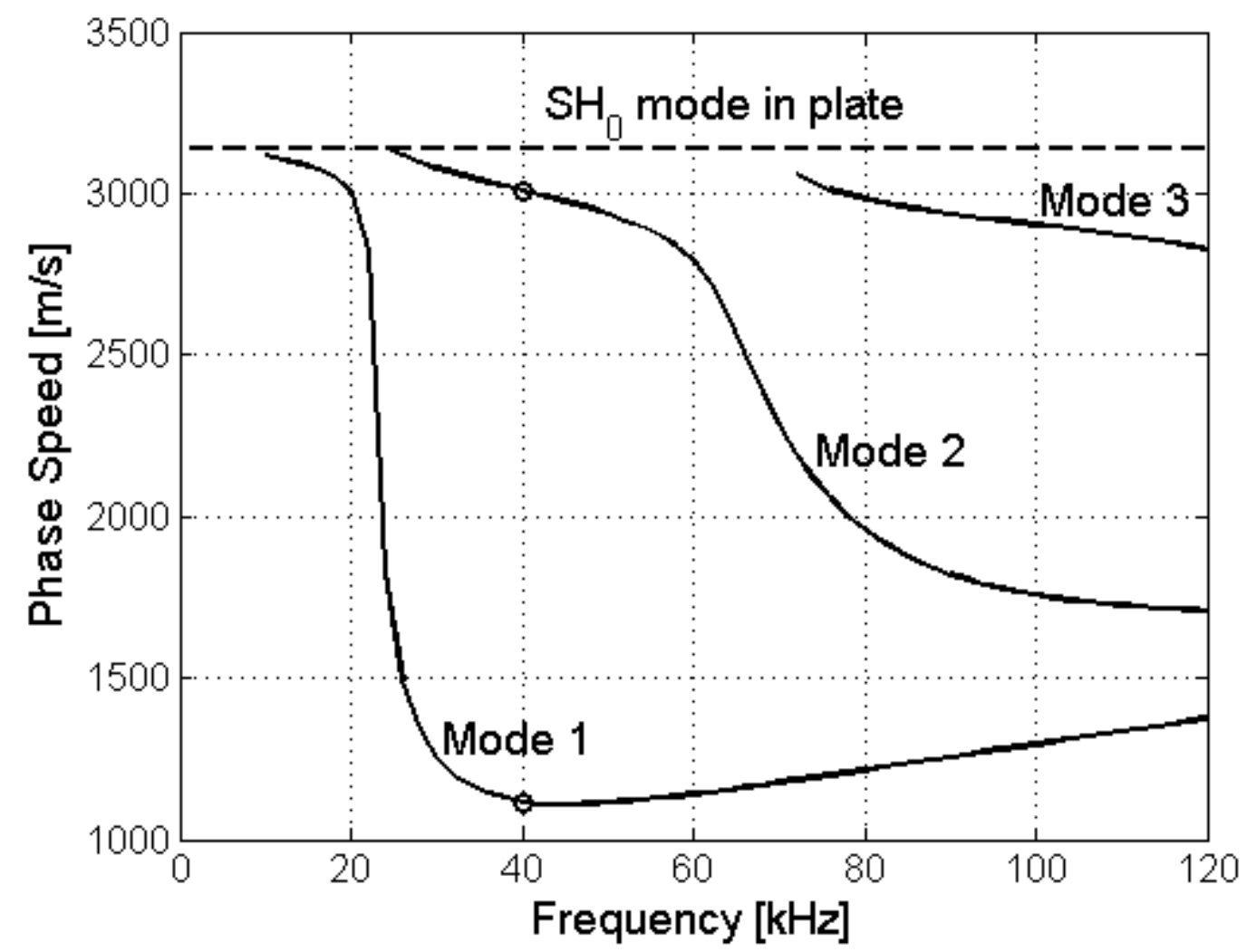

FIGURE 3. Phase speed of feature-guided shear waves propagating along stiffened plate calculated from SAFE model ( 3 modes, solid lines), bond line shear modulus $0.5 \mathrm{GPa}$, thickness $0.5 \mathrm{~mm}$; phase speed of $\mathrm{SH}_{0}$ mode in plate indicated (dashed line).
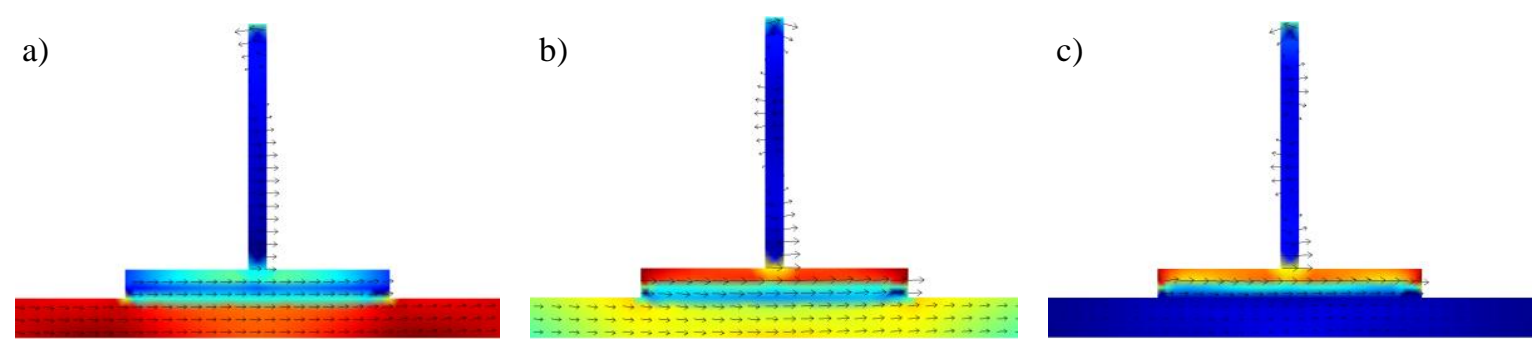

FIGURE 4. Mode shape illustration of feature-guided shear wave propagating along the stiffener, calculated from SAFE model at a) $\mathrm{f}=15 \mathrm{kHz}$, b) $\mathrm{f}=40 \mathrm{kHz}$, c) $\mathrm{f}=100 \mathrm{kHz}$; arrows depicting motion in waveguide cross-section. Color coding for each mode shows relative axial energy power flow (blue: low to red: high). 


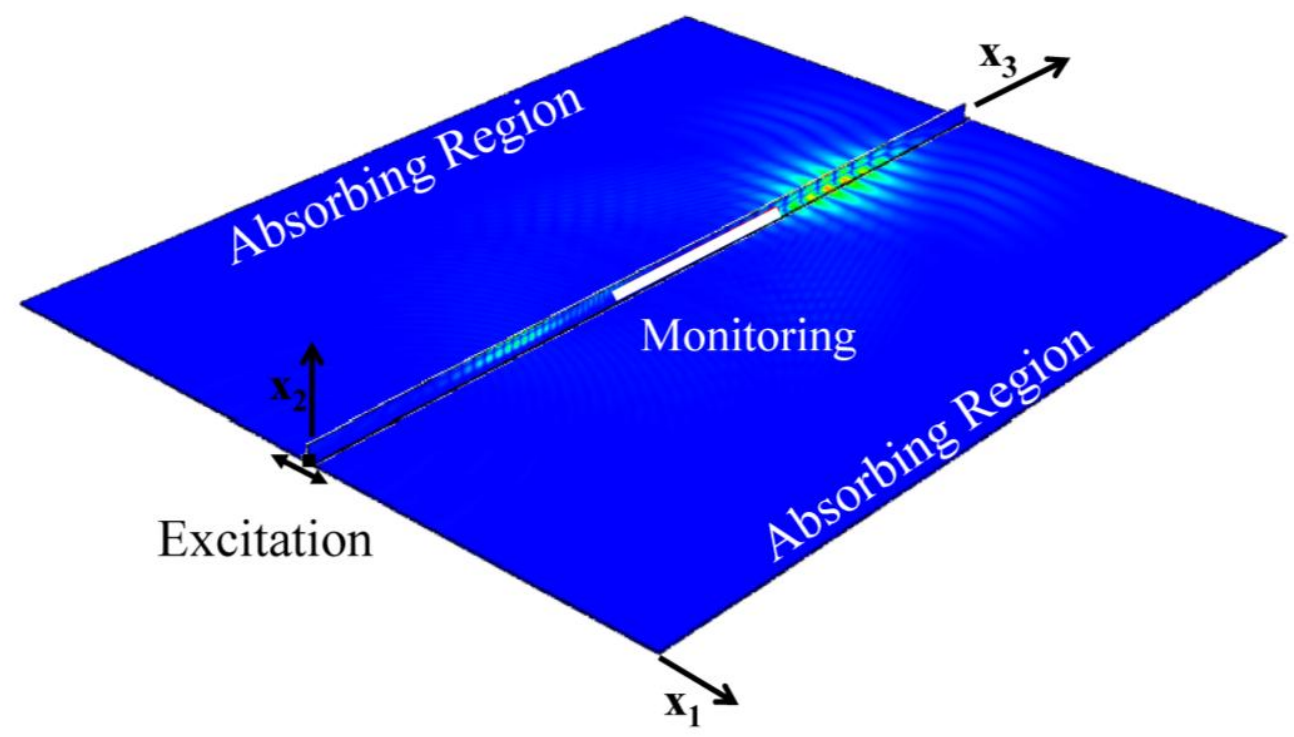

FIGURE 5. Schematic of 3D FE model of aluminium plate (size: $1000 \mathrm{~mm}$ x $1200 \mathrm{~mm}, 3 \mathrm{~mm}$ thickness) with T-shaped stiffener and adhesive bond; guided shear wave excitation at plate edge, monitoring locations and absorbing regions (width $400 \mathrm{~mm}$ ) indicated; colour coding shows relative amplitudes of propagating shear guided wave modes for $40 \mathrm{kHz}$ excitation frequency (blue: low to red: high).

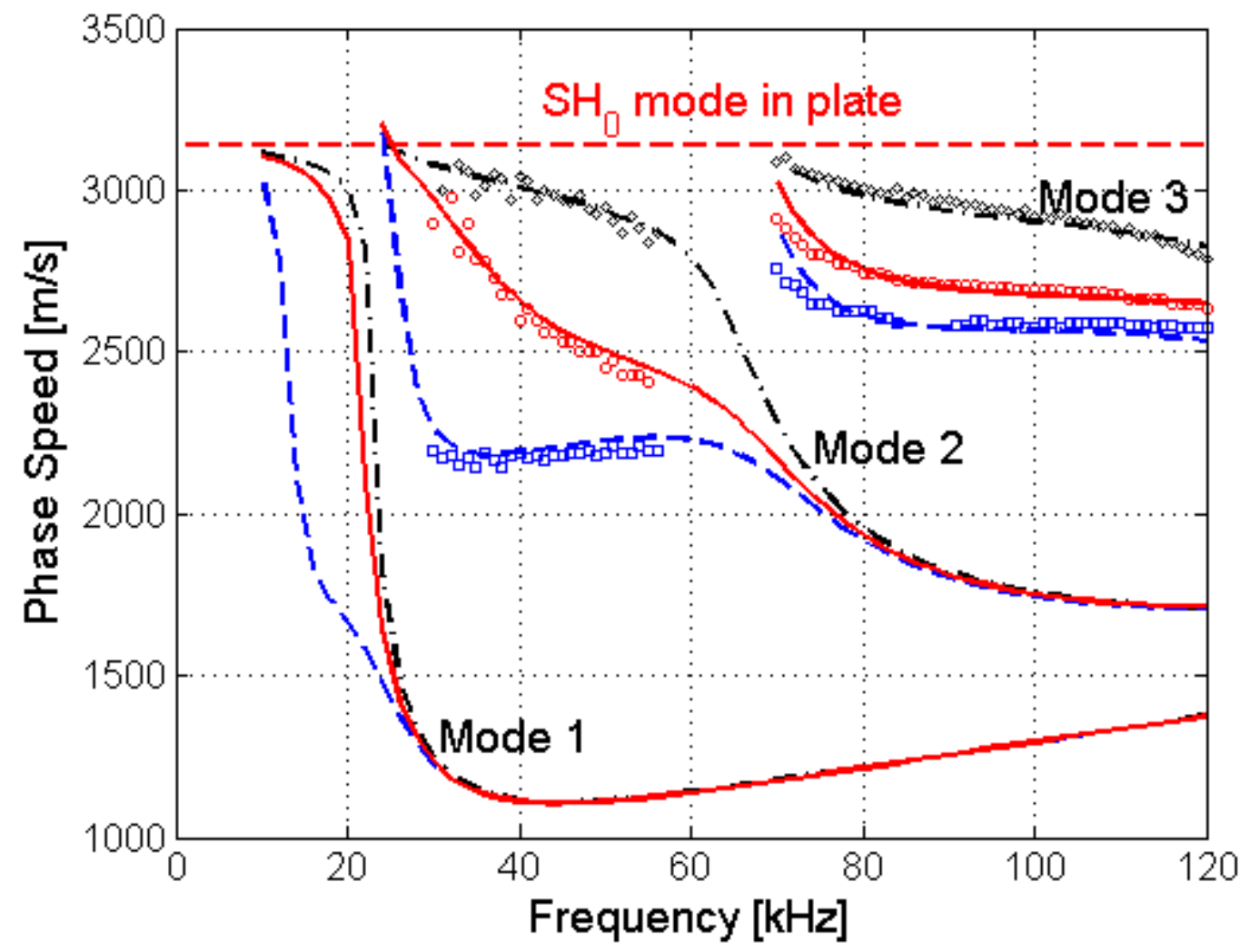

FIGURE 6. Effect of shear modulus of bond layer on phase speed dispersion curves. 3D FE simulations processed by 2D FFT: $\mathrm{G}=0.02 \mathrm{GPa}$ (blue, squares), $\mathrm{G}=0.1 \mathrm{GPa}$ (red, circles), $\mathrm{G}=0.5 \mathrm{GPa}$ (black, diamonds); SAFE predictions: $\mathrm{G}=0.02 \mathrm{GPa}$ (blue, dashed), $\mathrm{G}=0.1 \mathrm{GPa}$ (red, solid), $\mathrm{G}=0.5 \mathrm{GPa}$ (black, dash-dotted). Bondline thickness $0.5 \mathrm{~mm}$. 


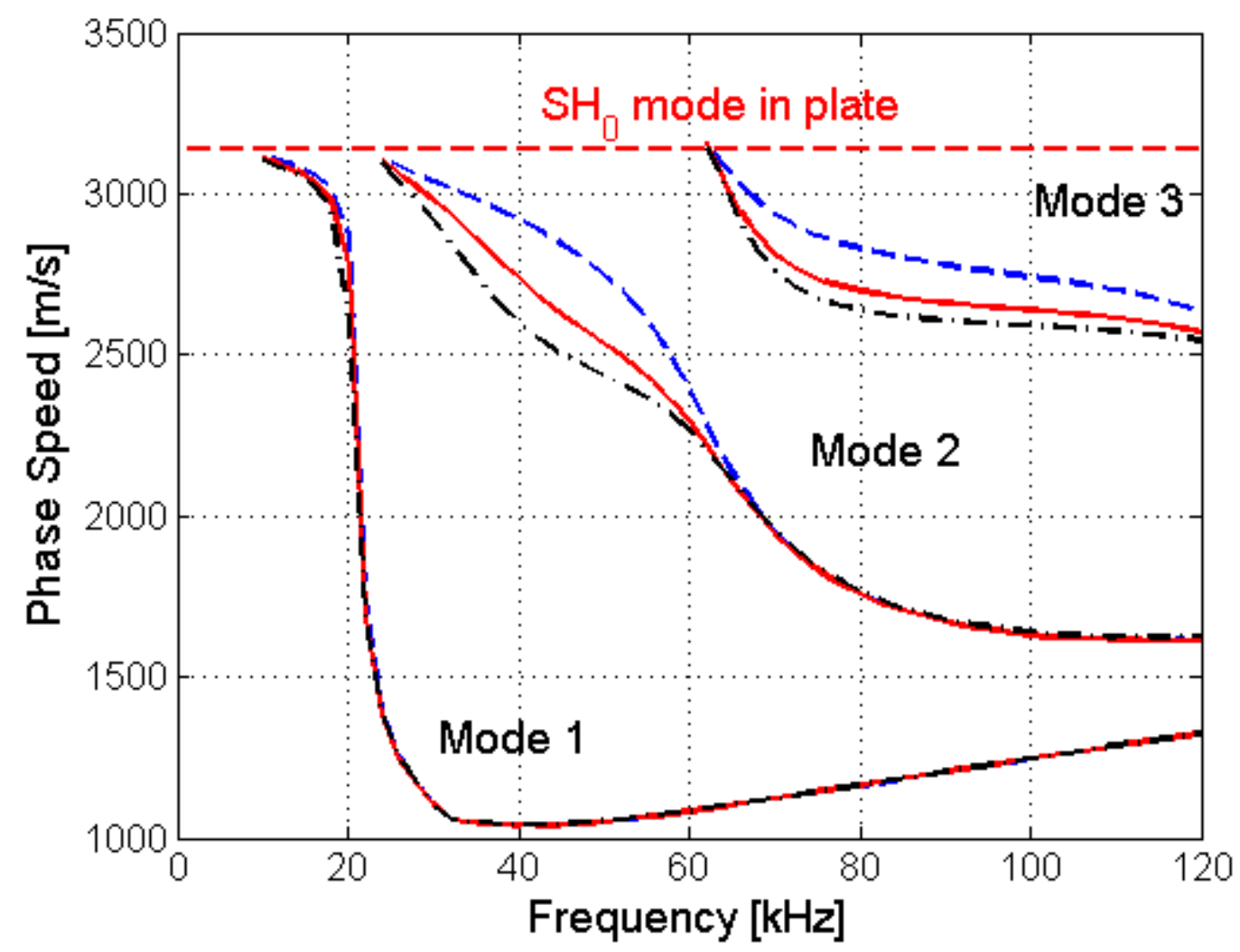

FIGURE 7. Effect of thickness of bond layer on phase speed dispersion curves, predicted by SAFE calculations: thickness $0.5 \mathrm{~mm}$ (blue, dashed), $0.8 \mathrm{~mm}$ (red, solid), $1.0 \mathrm{~mm}$ (black, dash-dotted). Bondline shear modulus $0.2 \mathrm{GPa}$.

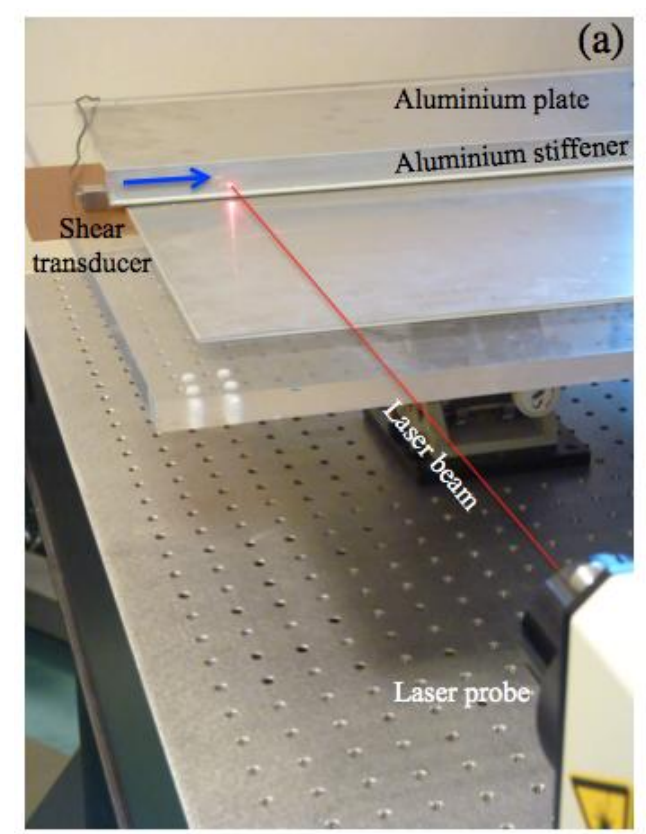

(b)

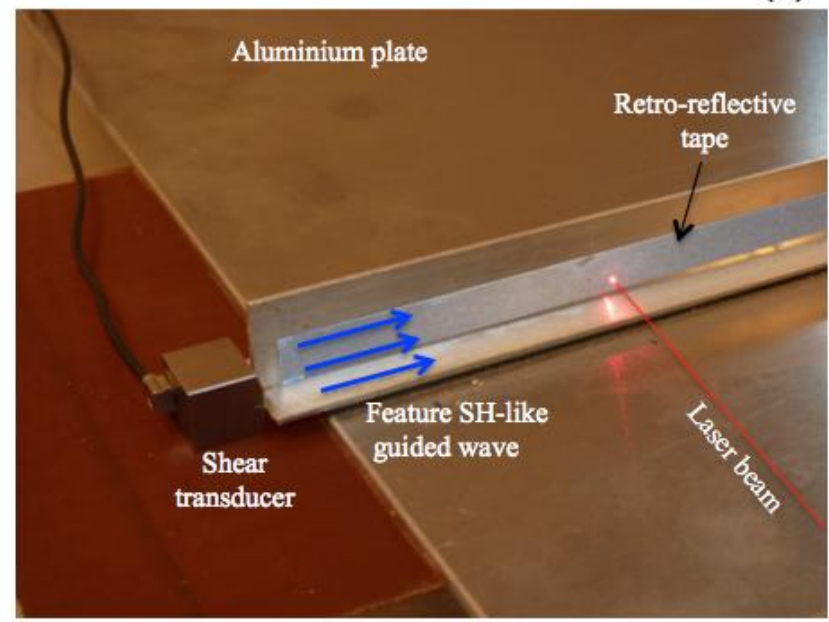

FIGURE 8. Experimental setup: a) aluminum plate with adhesively bonded stiffener, shear transducer, and laser interferometer (illustration) on optical table; b) detail of shear transducer excitation at end of stiffener, retro-reflective tape and illustration of laser beam. 


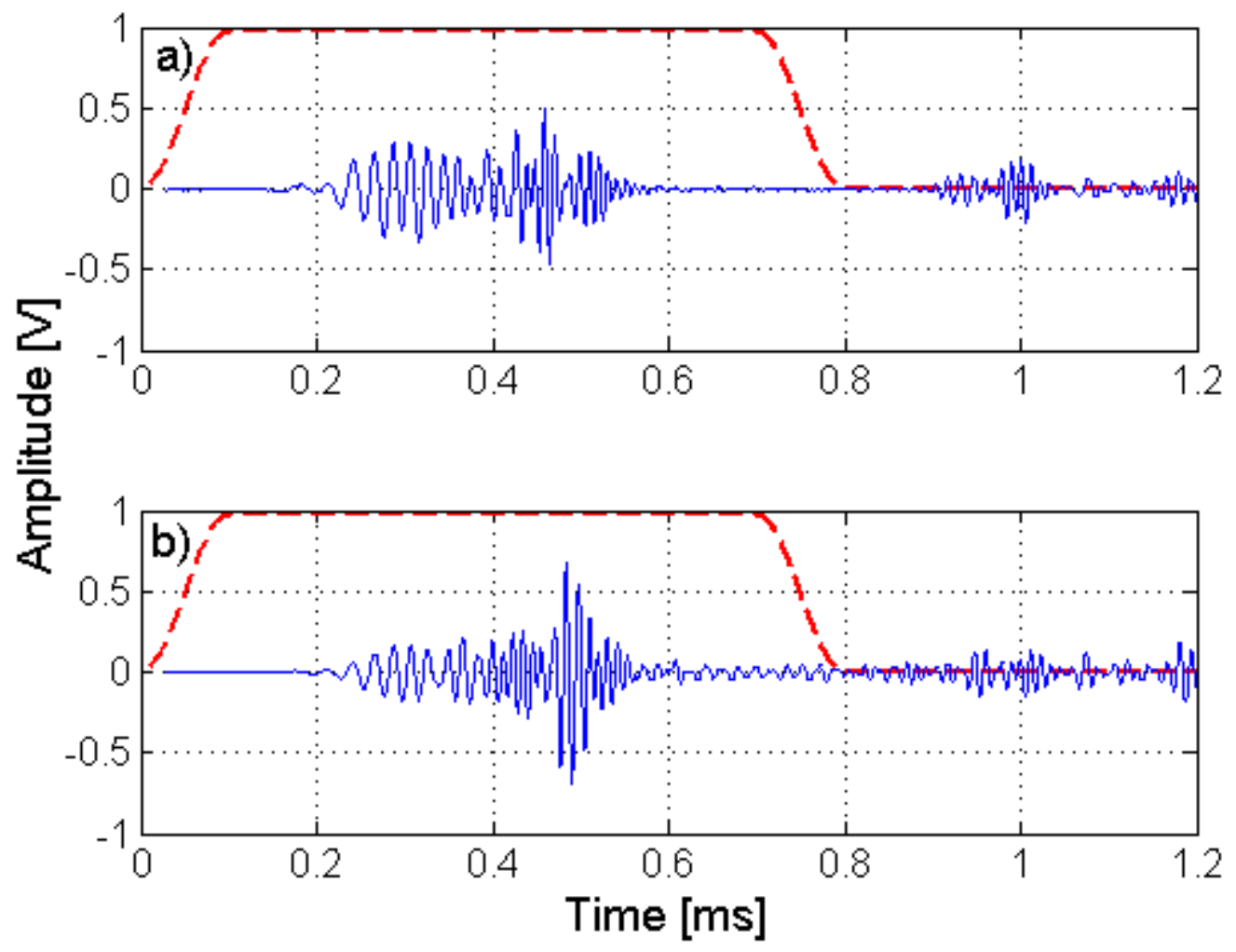

FIGURE 9. Typical experimental time traces with chosen time window, out-of-plane displacement on stiffener measured using laser interferometer, $170 \mathrm{~mm}$ from excitation; a) $3 \mathrm{~h}$ curing time; b) $164 \mathrm{~h}$ curing time.

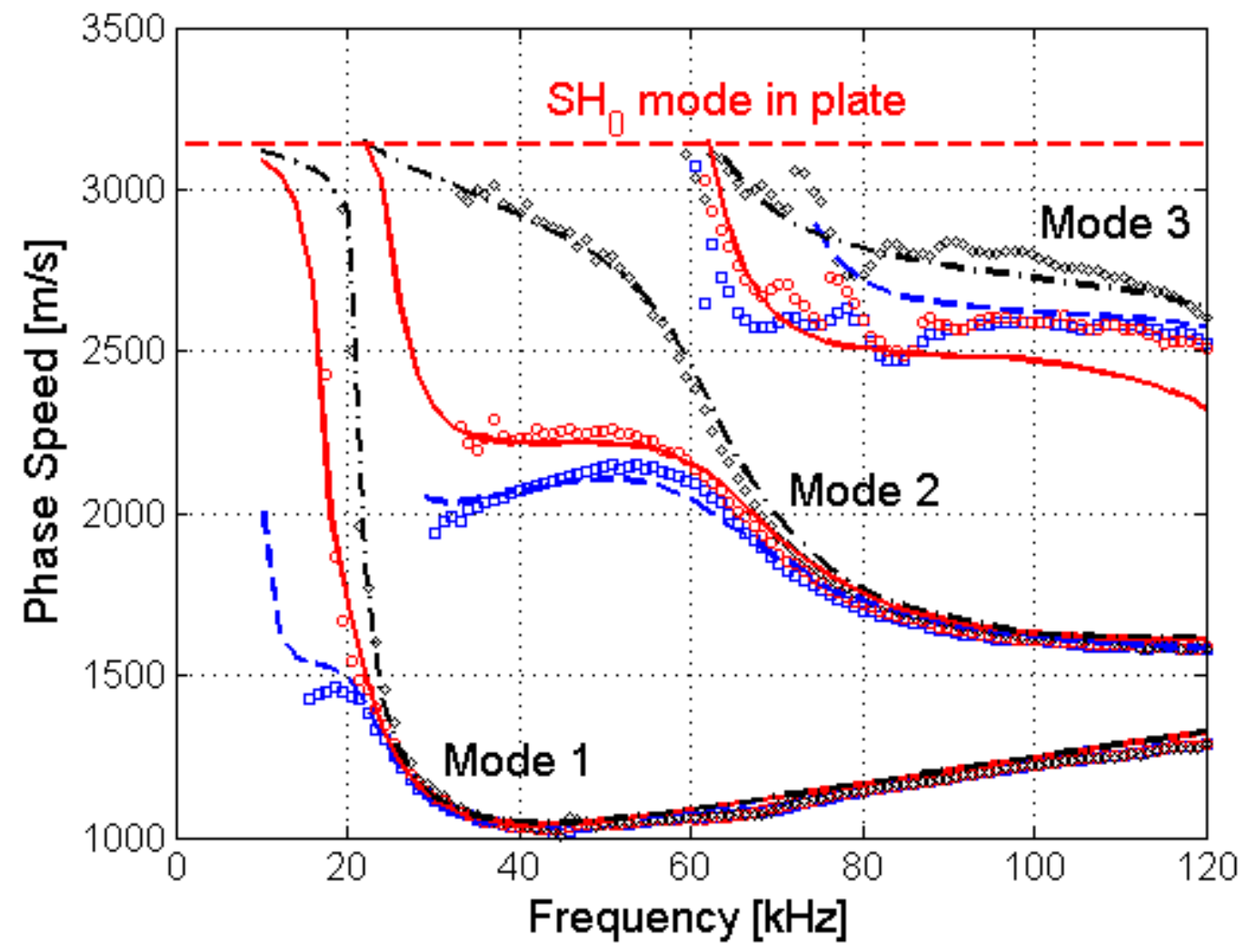

FIGURE 10. Experimentally measured phase speed dispersion curves during curing: $3 \mathrm{~h}$ curing time (blue, squares), $8 \mathrm{~h}$ curing time (red, circles), $164 \mathrm{~h}$ curing time (black, diamonds); comparison with SAFE 
predictions for best fit of bond layer shear modulus: $\mathrm{G}=0.02 \mathrm{GPa}$ (blue, dashed), $\mathrm{G}=0.07 \mathrm{GPa}$ (red, solid), $\mathrm{G}=0.5 \mathrm{GPa}$ (black, dash-dotted). Bondline thickness $1 \mathrm{~mm}$. 